Rev Inv Vet Perú 2016; ...(..): ...-... http://dx.doi.org/10.15381/rivep.

\title{
Comparación entre la Prueba de Puntas de Papel Absorbente Estériles y la Prueba Lagrimal de Schirmer para la Evaluación de Producción de Lágrimas en Canes Clínicamente Normales
}

\author{
Comparison between the Standardized Absorbent Paper Points Test and the \\ Schirmer's Tear Test to Evaluate Tear Production in Clinically \\ Healthy Dogs
}

Víctor Huaringa P. ${ }^{1,2}$, Ricardo Grandez R. ${ }^{1,3}$, Elizabeth Hinostroza M. ${ }^{1,4}$

\section{Resumen}

\begin{abstract}
El propósito del estudio fue determinar los valores normales de producción de lágrimas en perros mestizos, sin evidencias de alteraciones oftalmológicas, utilizando la prueba de puntas de papel absorbente estériles (PPPAE) y su comparación con la prueba lagrimal de Schirmer (PLS). Se evaluaron 33 perros (66 ojos) con cráneo de tipo dolicocéfalo, con edades de 2 a 6 años y de tamaño mediano ( $15 \mathrm{a} 24 \mathrm{~kg}$ ). El valor de la producción lagrimal promedio determinado mediante PPPAE fue de $20.85 \pm 1.44 \mathrm{~mm} / \mathrm{min}$ (rango de 18 a 24) y con la PLS de $18.05 \pm 1.58 \mathrm{~mm} / \mathrm{min}$. Se encontró una correlación positiva débil entre los resultados de PPPAE y PLS.
\end{abstract}

Palabras clave: puntas de papel absorbente estériles, prueba lagrimal de Schirmer, lágrimas, ojo

\section{AbStract}

The aim of the study was to determine normal values of tear production in crossbred dogs using the standardized absorbent paper points test (SAPPT) in comparison with the Schirmer's tear test (STT). A total of 33 dogs (66 eyes) of dolichocephalic skull type, between 2 to 6 years of age and of medium size $(15-24 \mathrm{~kg})$ were used. The mean tear

\footnotetext{
${ }^{1}$ Clínica Veterinaria Cayetano Heredia, Facultad de Medicina Veterinaria y Zootecnia, Universidad Peruana Cayetano Heredia, Lima, Perú

${ }^{2}$ E-mail: victor.huaringa@upch.pe

${ }^{3}$ E-mail: rgrandez@hotmail.com

${ }^{4}$ E-mail: clarisa.hinostroza@upch.pe
}

Recibido: 14 de octubre de 2014

Aceptado para publicación: 15 de marzo de 2015 
production value by SAPPT was $20.85 \pm 1.44 \mathrm{~mm} / \mathrm{min}$ (range: 18-24) and by STT was $18.05 \pm 1.58 \mathrm{~mm} / \mathrm{min}$. A positive weak correlation was found between SAPPT and STT values.

Key words: standardized absorbent paper points test, Schirmer's tear test, tear, eye

\section{INTRODUCCIÓN}

El sistema lagrimal canino está compuesto por un sistema excretor y uno secretor. El primero produce la película de lágrima preocular (PLP) y el segundo el drenaje desde el lado medial de la órbita para desembocar en la cavidad nasal (Brooks, 1992; Peiffer y Petersen-Jones, 2002; Petersen-Jones y Crispin, 2002; Stades, 2007).

La PLP está compuesta por una capa externa lipídica, una capa media acuosa y una capa interna mucosa. La capa lipídica, producida por las glándulas de Meibomio, estabiliza la película lagrimal y previene la evaporación de la capa acuosa. La capa acuosa es producida por la glándula lagrimal, accesoria lagrimal y nictitante, a fin de lubricar nutrir y proteger la córnea (Peiffer y Petersen-Jones, 2002; Broadwater et al., 2010; Turner, 2010). La capa mucosa es producida por las células caliciformes conjuntivales, la córnea y la conjuntiva, y su función es mantener la película lagrimal anclada al epitelio corneal, permitiendo que la capa acuosa se distribuya sobre la superficie, previniendo, a su vez, la desecación de la córnea y el daño del epitelio corneal durante el parpadeo (Petersen-Jones y Crispin, 2002; Davidson y Kuonen, 2004).

Una alteración de la película lagrimal puede desarrollar patologías relacionadas con insuficiencia de producción de lágrimas, sea cualitativamente como cuantitativamente, y con alteraciones en el drenaje de lágrimas, tanto por hiperproducción lagrimal o por obstrucción del drenaje (Peiffer y PetersenJones, 2002; Petersen-Jones y Crispin, 2002).
La queratoconjuntivitis seca (QCS) es una de las principales patologías diagnosticadas en pacientes caninos con problemas oftalmológicos. Su principal causa radica en una alteración cuantitativa de la PLP (Peiffer y Petersen-Jones, 2002; Petersen-Jones y Crispin, 2002; Westermeyer et al., 2009; Turner, 2010). También puede deberse a una alteración cualitativa de la PLP por alteraciones de producción de la capa lipídica o de la producción de mucina (Petersen-Jones y Crispin, 2002; Cunha, 2008; Turner, 2010). Las etiologías de estas alteraciones son de tipo medicamentosa, quirúrgica, idiopática, autoinmune, infecciosa y congénita, especialmente en razas como Buldog Inglés, West Highland, Cocker Spaniel, Dogo y Cavalier King Charles (Hartley et al., 2006; Cunha, 2008; Westermeyer et al., 2009; Turner, 2010).

Los tres métodos más usados en la práctica clínica para evaluar la producción de lágrimas son la prueba lagrimal de Schirmer (PLS), prueba lagrimal de Schirmer modificada (PLSM) y la prueba del hilo rojo de fenol (HRF).

La PLS es la prueba básica para la evaluación de la producción total de lágrimas (producción basal y refleja). Los valores normales en perros varían entre 15 a $25 \mathrm{~mm} / \mathrm{min}$ (Turner, 2010). Un estudio local con esta técnica reportó una media de $16.4 \pm 1.5 \mathrm{~mm} /$ min (Moreno et al., 2009). La PLS es eficiente en casos de exceso de producción lagrimal y déficit cuantitativo de producción (Westermeyer et al., 2009; Lange et al., 2012). La PLS modificada (PLSM) incluye el uso de anestesia tópica y permite evaluar 
solo la producción basal (Saito y Kotani, 2001; Hartley et al., 2006). Los valores normales de PLSM son más variables y en el nivel de $9.5 \pm 4.6 \mathrm{~mm} / \mathrm{min}$ (Saito y Kotani, 2001).

La prueba del HRF Se caracteriza por el cambio de color del reactivo de amarillo a rojo al contacto con las lágrimas, indicando la cantidad de producción de lágrimas. El rango de valores normales es de 24 a $35 \mathrm{~mm} /$ 15 s (Brooks, 1992; Saito y Kotani, 2001; Senchyna y Wax, 2008).

Los resultados de estas pruebas son afectadas por factores como raza, peso, edad, hora del día y múltiples patologías (distemper, seborrea generalizada, etc.) (Senchyna y Wax, 2008; Westermeyer et al., 2009; Broadwater et al., 2010; Turner, 2010). La PLS y PLSM presentan una baja reproductibilidad, alta variabilidad y baja correlación con los síntomas y signos causados por la insuficiencia de producción de lágrimas en comparación con HRF que presenta una mayor reproductibilidad y precisión en la evaluación de la producción de lágrimas (Senchyna y Wax, 2008). No obstante, la prueba del PLS es la más usada debido a su rigidez y por la dificultad de obtener los hilos de HRF, de producción japonesa (Senchyna y Wax, 2008).

En un estudio reciente sobre producción normal de lágrimas en primates Callithrix penicillata, se implementó un nuevo método para medir la capa acuosa. Se trata de la prueba de puntas de papel absorbente estériles (PPPAE) (Lange et al., 2012), las cuales destacan por su gran capacidad para absorber líquidos y mantener su rigidez al contacto con estos, así como una menor variación en los resultados. Por otro lado, el procedimiento es similar a PLS (ADA, 2008; Lange et al., 2012).

El presente estudio buscó establecer un rango de valores para PPPAE $(\mathrm{mm} / \mathrm{min})$ y determinar una posible correlación con la prueba lagrimal de Schirmer en perros mestizos sin evidencias de clínicas de alteraciones oftalmológicas.

\section{Materiales y Métodos}

El estudio se realizó en la Clínica Veterinaria Cayetano Heredia $(\mathrm{CVCH})$ de la $\mathrm{Fa}-$ cultad de Medicina Veterinaria y Zootecnia (FAVEZ) de la Universidad Peruana Cayetano Heredia (UPCH), entre diciembre de 2012 a enero de 2013.

\section{Animales}

Se determinó un tamaño de muestra mínimo de nueve perros, mediante la fórmula de tamaño muestral para poblaciones desconocidas, usando un nivel de confianza de $95 \%$ (Camacho-Sandoval, 2008). No obstante, se trabajó con 33 animales (17 hembras y 16 machos). Como criterios de inclusión se consideraron los perros atendidos en la $\mathrm{CVCH}$ entre las 08:00 y 12:00 horas, mestizos y de cráneo dolicocéfalo, con edades entre 2 a 6 años (adultos), de tamaño mediano (15 a 24 $\mathrm{kg}$ de peso vivo) y sin evidencias de alteraciones oftalmológicas al examen clínico general y regional oftalmológico (Tilley y Smith, 2011).

\section{Evaluación Ocular}

Se realizó una evaluación visual externa de córnea, pestañas, glándula nictitante, conjuntiva ocular y conducto naso-lagrimal para descartar posibles patologías o anormalidades (Turner, 2010). Los datos fueron recolectados en un formato clínico especialmente elaborado para este fin. Asimismo, se entregó una hoja de consentimiento informado al propietario, detallando los procedimientos a realizar para la autorización del reclutamiento del paciente.

\section{Evaluación de la Producción de Lágrima}

Se realizaron dos mediciones: la prueba de puntas de papel absorbente estériles (PPPAE) y la prueba lagrimal de Schirmer (PLS) como método de comparación en ambos ojos (tomados como muestras independientes), y con un intervalo de 10 min entre cada prueba (Yokoi et al., 2000). 


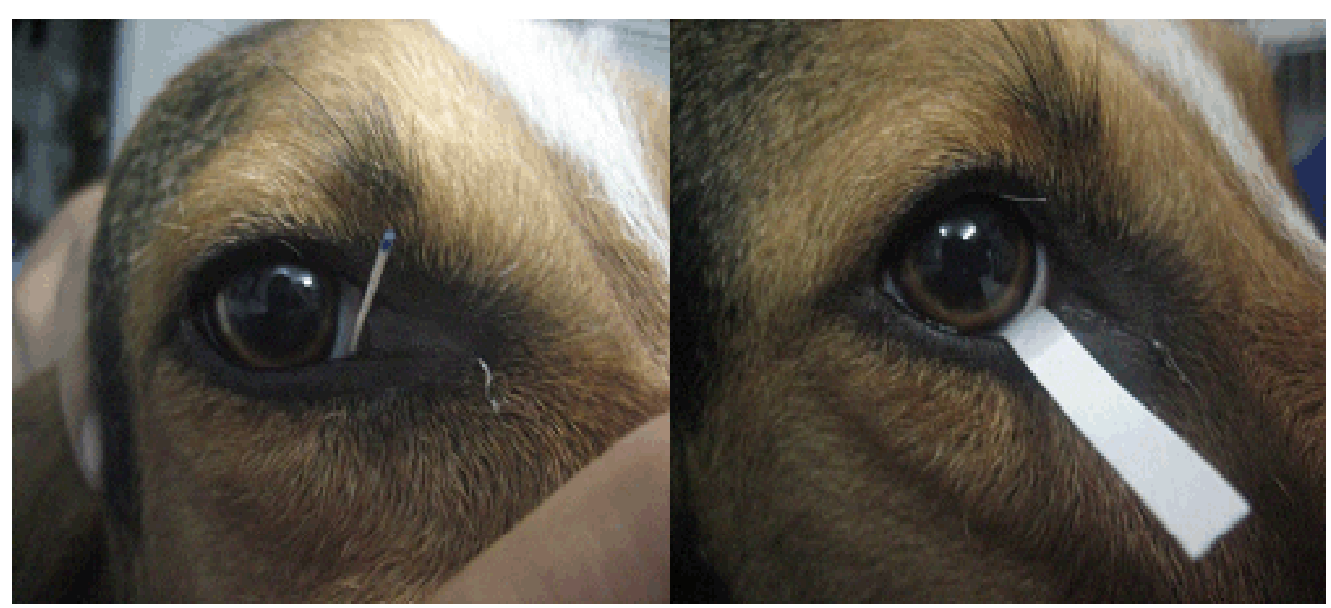

Figura 1. Vistas de la Prueba de Puntas de Papel Absorbente Estériles (PPPAE) y de la Prueba Lagrimal de Schirmer (PLS) en la medición de producción lagrimal en perros mestizos clínicamente normales

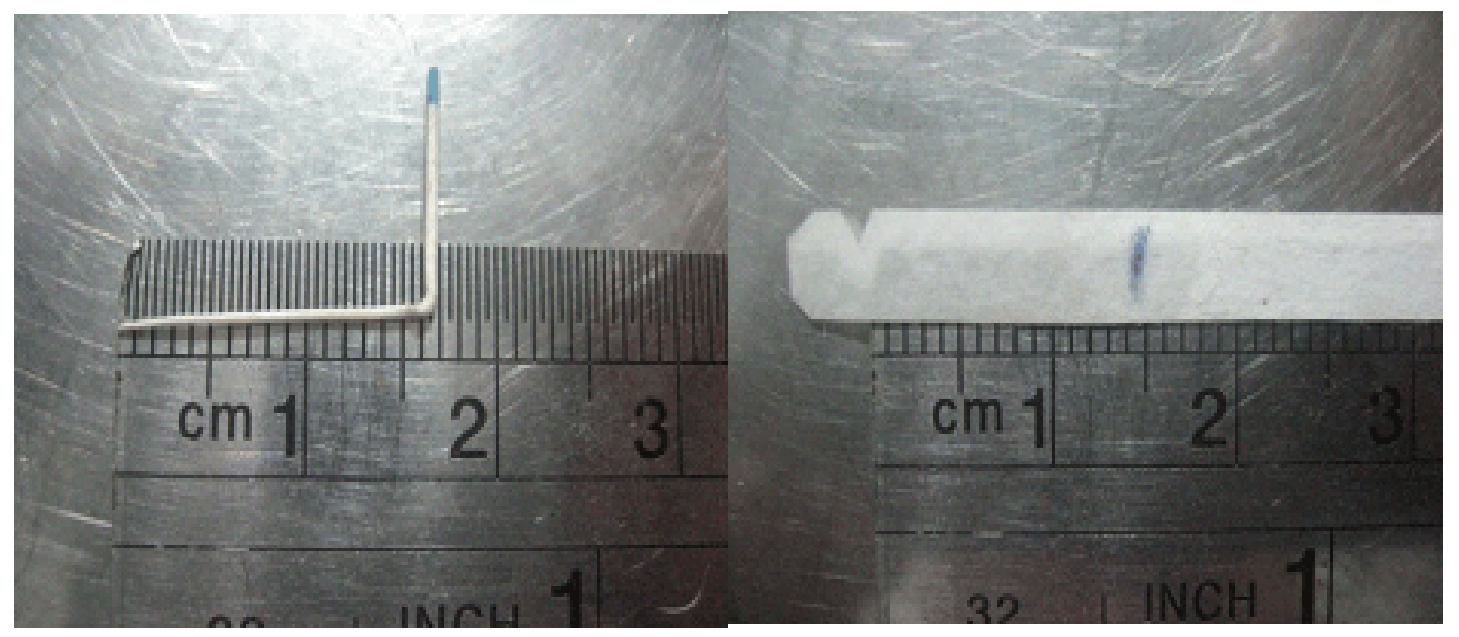

Figura 2. Vistas de la medición del recorrido de lágrima de la Prueba de Puntas de Papel Absorbente Estériles (PPPAE) y de la Prueba Lagrimal de Schirmer (PLS) en la medición de producción lagrimal en perros mestizos clínicamente normales. La porción final humedecida con lágrima se torna flexible y se dobla al ejercer presión contra una superficie dura

\section{Prueba de Puntas de Papel Absorbente Estériles (PPPAE)}

Se utilizaron puntas de papel absorbentes estériles Diadent, calibre 30 (Diamond Dental Industrial, Chongiu City, Korea), debido a la alta absorbencia mostrada en un estudio comparativo entre 12 marcas de pun- tas de papel absorbentes estériles (PumarolaSuñé, 1998). El extremo en punta fue introducido en el fórnix conjuntival inferior durante un minuto en cada ojo (Fig. 1). Se midió la parte húmeda con una regla milimetrada (Fig. 2), anotándose los valores en la hoja de formato clínico del paciente, según la metodología de Lange et al. (2012). 
Cuadro 1. Evaluación comparativa entre los resultados de la prueba lagrimal de Schirmer (PLS) y la Prueba de Puntas de Papel Absorbente Estériles (PPPAE) en la medición de la producción de lágrimas de 33 perros mestizos (66 ojos) clínicamente normales (diciembre 2012 - enero 2013, Lima, Perú)

\begin{tabular}{lccccccc}
\hline Prueba & $\begin{array}{c}\text { Muestras } \\
(\mathrm{n})\end{array}$ & $\begin{array}{c}\text { Media } \\
(\mathrm{mm} / \mathrm{min})\end{array}$ & $\begin{array}{c}\text { Mediana } \\
(\mathrm{mm} / \mathrm{min})\end{array}$ & $\begin{array}{c}\text { Desviación } \\
\text { estándar } \\
(\mathrm{mm} / \mathrm{min})\end{array}$ & $\begin{array}{c}\mathrm{CV}^{1} \\
(\%)\end{array}$ & $\begin{array}{c}\text { Mínimo } \\
(\mathrm{mm} / \mathrm{min})\end{array}$ & $\begin{array}{c}\text { Máximo } \\
(\mathrm{mm} / \mathrm{min})\end{array}$ \\
\hline $\begin{array}{l}\text { PLS } \\
(\mathrm{mm} / \mathrm{min})\end{array}$ & 66 & 18.05 & 18.00 & 1.38 & 0.08 & 15.00 & 21.00 \\
$\begin{array}{l}\text { PPPAE } \\
(\mathrm{mm} / \mathrm{min})\end{array}$ & 66 & 20.85 & 21.00 & 1.44 & 0.07 & 18.00 & 24.00 \\
\hline 1
\end{tabular}

Cuadro 2. Evaluación comparativa entre los resultados de cada ojo de la prueba lagrimal de Schirmer (PLS) y la Prueba de Puntas de Papel Absorbente Estériles (PPPAE) en la medición de la producción de lágrimas de 33 perros mestizos clínicamente normales (diciembre 2012 - enero 2013, Lima, Perú)

\begin{tabular}{cccccccc}
\hline Prueba & Ojo & $\begin{array}{c}\text { Media } \\
(\mathrm{mm} / \mathrm{min})\end{array}$ & $\begin{array}{c}\text { Mediana } \\
(\mathrm{mm} / \mathrm{min})\end{array}$ & $\begin{array}{c}\text { Desviación } \\
\text { estándar } \\
(\mathrm{mm} / \mathrm{min})\end{array}$ & $\begin{array}{c}\mathrm{CV}^{1} \\
(\%)\end{array}$ & $\begin{array}{c}\text { Mínimo } \\
(\mathrm{mm} / \mathrm{min})\end{array}$ & $\begin{array}{c}\text { Máximo } \\
(\mathrm{mm} / \mathrm{min})\end{array}$ \\
\hline $\begin{array}{c}\text { PLS } \\
(\mathrm{mm} / \mathrm{min})\end{array}$ & OD & 18.03 & 18.00 & 1.36 & 0.08 & 15.00 & 21.00 \\
OI & 18.08 & 18.00 & 1.42 & 0.08 & 15.00 & 21.00 \\
$(\mathrm{mPPAE} / \mathrm{min})$ & OD & 20.73 & 21.00 & 1.57 & 0.08 & 18.00 & 24.00 \\
\hline 1
\end{tabular}

${ }^{1}$ Coeficiente de variación

Cuadro 3. Evaluación comparativa entre los resultados por sexo (16 machos y 17 hembras) de la prueba lagrimal de Schirmer (PLS) y la Prueba de Puntas de Papel Absorbente Estériles (PPPAE) en la medición de la producción de lágrimas de 66 ojos de 33 perros mestizos clínicamente normales (diciembre 2012 - en ero 2013, Lima, Perú)

\begin{tabular}{clcccccc}
\hline Prueba & Sexo & $\begin{array}{c}\text { Media } \\
(\mathrm{mm} / \mathrm{min})\end{array}$ & $\begin{array}{c}\text { Mediana } \\
(\mathrm{mm} / \mathrm{min})\end{array}$ & $\begin{array}{c}\text { Desviación } \\
\text { estándar } \\
(\mathrm{mm} / \mathrm{min})\end{array}$ & $\begin{array}{c}\mathrm{CV}^{1} \\
(\%)\end{array}$ & $\begin{array}{c}\text { Mínimo } \\
(\mathrm{mm} / \mathrm{min})\end{array}$ & $\begin{array}{c}\text { Máximo } \\
(\mathrm{mm} / \mathrm{min})\end{array}$ \\
\hline $\begin{array}{c}\text { PLS } \\
(\mathrm{mm} / \mathrm{min})\end{array}$ & Macho & 17.96 & 18.00 & 1.67 & 0.09 & 15.00 & 21.00 \\
& Hembra & 18.16 & 18.00 & 0.99 & 0.05 & 16.00 & 20.00 \\
PPPAE & Macho & 20.65 & 21.00 & 1.25 & 0.06 & 18.00 & 23.00 \\
$(\mathrm{~mm} / \mathrm{min})$ & Hembra & 21.06 & 21.00 & 1.61 & 0.08 & 18.00 & 24.00 \\
\hline
\end{tabular}

${ }^{1}$ Coeficiente de variación 


\section{Prueba Lagrimal de Schirmer (PLS)}

Se utilizaron tiras de Test de Schirmer (Laboratorio Love Sudamericana, Buenos Aires Argentina). Se siguieron las indicaciones de Turner (2010), plegando cada tira por la muesca y ubicando en la parte media del párpado inferior de cada ojo durante un minuto (Fig. 1). La medida de la distancia desde el doblez de la muesca hasta el extremo humedecido de la tira fue tomada con una regla milimetrada (Fig. 2) Los valores se anotaron en la hoja de formato clínico del respectivo paciente.

\section{Análisis de Datos}

Los datos fueron analizados con al programa estadístico R (R-Core-Team, 2012). Además, se realizó el Coeficiente de Correlación de Pearson entre los valores de PLS y PPPAE para cada ojo y por sexo para determinar posibles asociaciones. Se hizo el Test de Student Pareado y el Test de Student No Pareado, según el caso, para determinar diferencias estadísticas entre las variables PLS, PPPAE y sus valores para cada ojo y sexo.

\section{Resultados}

La media para PLS fue de $18.05 \pm 1.38$ $\mathrm{mm} / \mathrm{min}$ y para PPPAE de $20.85 \pm 1.44 \mathrm{~mm} /$ min. En el Cuadro 1 se muestra el análisis descriptivo de los resultados para esta prueba. Los valores entre ojo derecho e izquierdo presentaron una correlación positiva fuerte para ambas pruebas $(\mathrm{r}=0.7553$ para PLS y $\mathrm{r}=0.7720$ para PPPAEA). Por otro lado, no se observó diferencia significativa entre la producción lagrimal entre ojos $(\mathrm{p}=0.7898$ para PLS y $\mathrm{p}=0.1737$ para PPPAE) ni entre sexos $(p=0.553$ para PLS y $p=0.3218$ para PPPAEA) (Cuadros 2 y 3 ).

El coeficiente de correlación de Pearson mostró correlaciones positivas débiles entre las variables PLS y PPPAE $(r=0.4743978)$, PLS y PPPAE para el ojo derecho $(\mathrm{r}=0.3713)$ y para el ojo izquierdo $(r=0.5995)$, y PLS y PPPAE para hembras $(\mathrm{r}=0.4917)$ y para machos $(\mathrm{r}=0.5355)$.

Al realizar la prueba de Test de Student se halló diferencia estadística entre los resultados de PLS y PPPAE $(\mathrm{p}=0.0000)$, PLS y PPPAE para el ojo derecho $(p=0.0000)$ e izquierdo $(\mathrm{p}=0.0000)$, y PLS y PPPAE para hembras $(p=0.0000)$ y machos $(p=0.0000)$.

\section{Discusión}

El valor promedio de producción de lágrimas hallado con la PLS para perros mestizos se encuentra dentro del rango descrito por otros autores (Saito y Kotani, 2001; Turner, 2010). Asimismo, no hubo diferencias entre los valores de PLS de ojo derecho e izquierdo, mostrando similitudes con otros resultados (Hartley et al., 2006; Moreno et al., 2009).

La producción promedio de lágrimas hallada con la PPPAE de $20.85 \pm 1.44 \mathrm{~mm} /$ min, y sin diferencia estadística entre ojos derecho e izquierdo, será de utilidad en futuros estudios con esta técnica en perros u otras especies animales. Actualmente no existen valores de referencia en perros usando esta prueba.

La ausencia de diferencias entre ojo derecho e izquierdo era esperado (Turner, 2010), debido a que los perros no presentaban ningún tipo de afección ocular, y el parpadeo ocurría al mismo tiempo en ambos, de allí que la distribución de la película lagrimal era similar en ambos ojos.

Las medias de producción lagrimal medidas mediante PPPAE y PLS fueron diferentes, tanto en medidas promedio generales, como por ojo y por sexo. Esto puede ser ocasionado por las diferencias entre los materiales utilizados. En el caso de la PPPAE se emplean conos enrollados de papel de 28 $\mathrm{mm}$ de largo y con un calibre de $30 \mathrm{G}(0.3$ 
$\mathrm{mm})$, en tanto que las tiras de papel de la PLS miden $5 \mathrm{~mm}$ de ancho por $50 \mathrm{~mm}$ de largo, causando en este último caso un menor avance en milímetros por minuto debido a la mayor superficie de absorción.

Las mediciones con la PPPAE fueron relativamente más fáciles de realizar, ya que en algunos casos se tuvo que realizar más de un intento con la PLS, posiblemente debido a la irritación causada al globo ocular con la tira de papel (Senchyna y Wax, 2008).

Con base a los resultados del presente estudio, se recomienda realizar estudios en perros con patologías oftálmicas y, de esta forma, poder validar el uso de la PPPAE como prueba de rutina en la evaluación ocular de pacientes en la práctica clínica.

\section{Literatura Citada}

1. [ADA] American Dental Association. 2008. ANSI/ADA Standard No. 73 Dental absorbent points: 2008 (Reaffirmed 2013). [Internet]. Disponible en: http://www.ada.org/830.aspx\#73

2. Broadwater JJ, Colitz C, Carastro S, Saville $W$. 2010. Tear production in normal juvenile dogs. Vet Ophthalmol 13: 321-325. doi: 10.1111/j.1463-5224.2010. 00820.x

3. Brooks DE. 1992. Conceptos Actuales en Oftalmología Veterinaria: Colegio Estadounidense de Oftalmología Veterinaria. [Internet]. Disponible en: http:// www.vetmed.ufl.edulsacs/ophtho/ 04amveppanotesspanish.pdf

4. Camacho-Sandoval J. 2008. Tamaño de muestra en estudios clínicos. Acta Méd Costarric 50: 20-21.

5. Cunha O. 2008. Manual de oftalmologia veterinaria. Brasil: Universidade Federal Do Paraná. 88 p.

6. Davidson HJ, Kuonen VJ. 2004. The tear film and ocular mucins. Vet Ophthalmol 7: 71-77. doi: 10.1111/j.14635224.2004.00325.x
7. Hartley C, Williams DL, Adams VJ. 2006. Effect of age, gender, weight, and time of day on tear production in normal dogs. Vet Ophthalmol 9: 53-57.

8. Lange RR, Lima L, MontianiFerreira F. 2012. Measurement of tear production in black-tufted marmosets (Callithrix penicillata) using three different methods: modified Schirmer's I, phenol red thread and standardized endodontic absorbent paper points. Vet Ophthalmol 15: 376-82. doi: 10.1111/ j.1463-5224.2012.00998.x

9. Moreno J, Fernández V, Chipayo Y, Crespo A. 2009. Patrones referenciales de producción lacrimal en caninos, empleando la prueba de Schirmer, en una clínica de Lima Metropolitana. Rev Inv Vet, Perú 20: 249-253. doi: 10.15381/ rivep.v20i2.618

10. Peiffer RL, Petersen-Jones S. 2002. Oftalmología de pequeños animales: un enfoque orientado a la resolución de problemas. $3^{\mathrm{a}}$ ed. España: Elsevier. 266 p.

11. Petersen-Jones S, Crispin S. 2002. BSAVA Manual of small animal ophthalmology. $2^{\text {nd }}$ ed. UK: British Small Animal Veterinary Association. 324 p.

12. Pumarola-Suñé J, Solá-Vicens L, Sentís-Vilalta J, Canalda-Sahli C, BrauAguadé E. 1998. Absorbency properties of different brands of standardized endodontic paper points. J Endodont 24: 796-798. doi: 10.1016/ S0099-2399(98)80004-6

13. R-Core-Team. 2012. R: A language and environment for statistical computing. Ed 2.15.1. Vienna, Austria: R Development Core Team. 3604 p.

14. Saito A, Kotani T. 2001. Estimation of lacrimal level and testing methods on normal beagles. Vet Ophthalmol 4: 7-11. doi: 10.1046/j.1463-5224.2001.00124.x

15. Senchyna M, Wax MB. 2008. Quantitative assessment of tear production: A review of methods and utility in dry eye drug discovery. J Ocul Biol Dis Infor 1(1): 1-6. doi: 10.1007/ s12177-008-9006-2 
16. Stades FC, Wyman M, Boeve MH, Neumann W, Spiess, B. 2007. Ophthalmology for the veterinary practitioner. $2^{\text {nd }}$ ed. Germany: Schlütersche Verlagsgesellschaf. $204 \mathrm{p}$.

17. Tilley LP, Smith FWK. 2011. Blackwell's five-minute veterinary consult: canine and feline. USA: WileyBlackwell. 1600 p.

18. Turner RM. 2010. Oftalmología de pequeños animales. España: Elsevier. 384 p.
19. Westermeyer HD, Ward DA, Abrams K. 2009. Breed predisposition to congenital alacrima in dogs. Vet Ophthalmol 12: 1-5. doi: 10.1111/j.14635224.2009.00665.x.

20. Yokoi N, Kinoshita S, Bron AJ, Tiffany JM, Sugita J, Inatomi T. 2000. Tear meniscus changes during cotton thread and Schirmer testing. Invest Ophthalmol Vis Sci 41: 3748-3753. 